\section{S03.2 GLOBAL AND REGIONAL ESTIMATES OF HSV2, HSV1 AND NEONATAL HERPES FOR 2012}

Katherine Turner*. University of Bristol, Bristol, UK

\subsection{6/sextrans-2015-052270.28}

Background Genital infection with herpes simplex virus type 2 (HSV-2) is the most common cause of genital ulcer disease worldwide and increases susceptibility to, and transmissibility of, HIV. HSV type 1 (HSV-1) can also cause genital herpes, but most HSV-1 infection is oro-labial ("cold sores") and acquired during childhood. Neonatal herpes, acquired during delivery from mothers with genital herpes, is rare but has high rates of mortality and lifelong disability. The global burden of HSV-2 infection was last estimated for 2003. The global burden of HSV-1 infection, and of neonatal herpes, has never been estimated to our knowledge.

Aim To present, for 2012, new global HSV-2 estimates for females and males aged 15-49 years, first global HSV-1 estimates for females and males aged 0-49 years, and first global estimates of neonatal herpes cases.

Methods Literature review of recent HSV-1 and HSV-2 prevalence studies world-wide, followed by fitting of a model with constant incidence by age to pooled prevalence values by HSV type, WHO region, age and sex. Prevalence values were adjusted for test sensitivity and specificity. Published risks of neonatal herpes transmission were applied to maternal infections to estimate neonatal herpes cases.

Results We estimate that in 2012, 417 million people aged 1549 years (range: 274-678 million) had existing HSV-2 infection world-wide: a global prevalence of $11.3 \%$. Of those infected, 267 million were women. Also in 2012, we estimate that 19.2 million (range: 13.0-28.6 million) individuals aged 15-49 years were newly-infected with HSV-2: $0.5 \%$ of all individuals globally. Prevalence was highest in Africa (31.5\%), followed by the Americas (14.4\%). Burden of numbers infected was highest in Africa. However, despite lower prevalence, South-East Asia and Western Pacific regions also contributed large numbers to the global totals because of large population sizes. Estimates for HSV-1 and neonatal herpes will be presented at the conference. Conclusions The global burden of HSV infection is large. This highlights the critical need for development of vaccines, microbicides and other prevention strategies against HSV.

\section{S03.3 STI ESTIMATES WITHIN THE INSTITUTE FOR HEALTH METRICS AND EVALUATION GLOBAL BURDEN OF DISEASE PROCESS}

Nick Kassebaum*. Institute for Health Metrics and Evaluation, Seattle, USA

10.1136/sextrans-2015-052270.29

Introduction Sexually-transmitted infections (STIs) are an important cause of acute illness, disability and death throughout the world. In this study, we used GBD 2013 systematic approach to analyze levels and trends in health loss due to STI for the years 1990 to 2013, for 188 countries, both sexes, and 20 separate age groups. We separately estimated deaths, years of life lost (YLLs), prevalence, incidence and years of life lived with disability (YLDs) for five different infections: gonorrhea (Neisseria gonorrhea), chlamydia (Chlamydia trachomatis), trichomoniasis (Trichomonas vaginalis), syphilis (Treponema pallidum), genital herpes due to herpes simplex virus 2 (HSV-2), and the residual category of "other" STI.

Methods Age- and sex-specific mortality due to all STI in ages 10 and over was estimated using cause-of-death ensemble modeling (CODEm). Syphilis deaths in ages 0 to 9 used a natural history model combining data on syphilis prevalence in pregnancy, live births, antenatal care coverage, routine antenatal syphilis testing, and excess neonatal mortality rates in those infected with syphilis. Years of life lost (YLL) were calculated by multiplying age-specific deaths with life expectancy at time of death. We estimated non-fatal STI burden consequent to acute, recurrent, and chronic infection as well as pelvic inflammatory disease (PID) and infertility. Our dataset was developed via systematic literature review, supplemented with national reports and hospital datasets. We modeled the epidemiology of each condition using DisMod-MR 2.0, a Bayesian meta-regression tool developed for GBD 201313, thereby generating age- and sex-specific prevalence and incidence estimates for each condition. Years of life lived with disability (YLD) were calculated by pairing symptomatic case numbers with corresponding disability weights (DW).

Results Global STI deaths decreased from 257,648 (95\% UI: $154,732$ to 396,443$)$ in 1990 to $142,017(87,589$ to 213,920$)$ in 2013. Most deaths were due to congenital syphilis which was the dominant driver of YLLs. Over 99\% of all STI deaths occurred in developing countries, including 32.9\% in eastern sub-Saharan Africa alone. Annualised rate of change (ARC) of age-standardized death rates globally was $-3.0 \%$, with 38 countries exceeding $-5.0 \%$. New cases of chlamydia, gonorrhea, trichomoniasis and genital herpes all increased between 1990 and 2013 and were amongst the top 25 most common acute conditions. North Africa and the Middle East saw the biggest percentage increase, driven mostly by population growth. Peak ages for incident infection in each STI were between 20 and 25 years and largely unchanged from 1990 to 2013. The most common acute STI in females was trichomoniasis whereas it was chlamydia in males. Genital herpes was by far the most common chronic infection in both sexes. In females, chlamydia and gonorrhea were the dominant driver of YLDs, mostly due to PID and infertility, whereas genital herpes was the biggest cause of disability due to STI in males.

Discussion Despite significant improvement in congenital syphilis mortality from 1990 to 2013, STI remain a significant cause of acute infection and disability throughout much of the world, even increasing in many locations. Surveillance and treatment programs for STI should remain a priority.

\section{SO3.4 FUTURE DIRECTIONS FOR GLOBAL STI ESTIMATES}

Teodora Wi*. World Health Organization, Geneva, Switzerland

\subsection{6/sextrans-2015-052270.30}

Background Estimating STI prevalence and incidence are important for programming and advocacy. Current global and regional estimates are based on meta-analysis of prevalence data of gonorrhoea, chlamydia and trichomoniasis among low-risk populations, and on national surveillance of syphilis sero-prevalence among antenatal care attendees, with adjustment for laboratory test types, geography and age. The precision and utility of these estimates is limited by insufficient, varying, and uncertain data availability and quality, notably on duration of infection and symptoms, representativeness of survey samples, etc. Current 